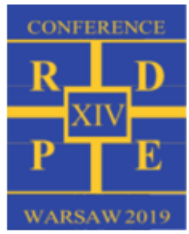

\title{
Critical review and economic feasibility analysis of electric energy storage technologies suited for grid scale applications
}

\author{
Guido Francesco Frate $^{1, *}$, Lorenzo Ferrari ${ }^{2}$, and Umberto Desideri ${ }^{3}$ \\ ${ }^{1}$ U niversity of Pisa, V ia Largo Lucio Lazzarino 1, 56122 - Pisa, guidofrancesco.frate@ ing.unipi.it, I taly \\ 2 University of Pisa, Via Largo L ucio Lazzarino 1, 56122 - Pisa, Iorenzo.ferrari@ unipi.it, Italy \\ 3 University of Pisa, Via Largo Lucio Lazzarino 1, 56122 - Pisa, umberto.desideri@ unipi.it, Italy
}

\begin{abstract}
Radical changes in production paradigms are affecting electric systems. This is due to Renewable Energy Sources (RES) introduction, which changed production from being centralized and dispatchable, to be more decentralized and unpredictable. Further transformations are expected as the RES share into electric grids will increase. RES further development will likely rely mostly on additional solar PV capacity, then relevant energy quantities will be needed to be shifted from day hours to evening and night hours. Such "Load Shifting" could be performed with Electric Energy Storage (EES) technologies. Few technologies suited for this are al ready commercially available, whereas others have been proposed. In this paper, the EES technologies suited for load shifting are reviewed with a focus on economic costs. A fter that, current and future EES economic feasibility are assessed by using Italian hourly energy prices from 2018. Since EES resulted to be currently uneconomic, the minimum price modification required to make EES feasible is calculated.
\end{abstract}

\section{Introduction}

Nowadays, electric systems are changing due to nondispatchable RES introduction, mostly wind energy and solar PV. Since non-dispatchable RESs are rigid energy sources, their impact is making the grids less flexible. Furthermore, RESs are prioritized on energy markets, hence they are superseding traditional power plants. These are forced to provide the additional flexibility required by non-dispatchable RES by operating in part load and by doing frequent start-ups [1]. Storage might partly solve these issues, because it may smooth RES fluctuations, thus reducing traditional power plant need for part loads and start-ups. Unfortunately, suited sites for Pumped Hydro Energy Storage (PHES) are already exploited [2] and no new storage technologies have been deployed to date. In this situation, as non-dispatchable RESs grow, electric systems might become more and more rigid, up to the point of lacking the flexibility required to be safely operated.

Since most of RES additional capacity will likely come from solar PV [3], traditional power plants might be forced to fast ramping during evening hours to replace solar production. Traditional power plants could be supported in this task by EESs, which could shift energy from daytime to evening. To do that, storages that are able to charge at full power for $4 / 6$ hours to absorb energy during solar production peaks might be required [4]. Such EESs would be characterized by a power/capacity ratio equal to $1 / 6-1 / 4 h^{-1}$. However, not all the EESs may achieve this configuration efficiently from the economic point of view, since their capacityrelated costs are higher than their power-related ones. Current EESs suited for this are: PHES, Compressed A ir
Energy Storage (CAES), Liquified Air Energy Storage (LAES), Pumped Thermal Energy Storage (PTES), molten salt $(\mathrm{NaS})$ batteries and Flow batteries. These technologies are here briefly reviewed. By focusing on the economic aspects, current and future economic feasibility of such EESs is assessed. In conclusion, the main contributions of the present analysis are:

- an EES technology review is performed, by including also some often-overlooked technologies like PTES and PHES-based systems like Sea Water PHES (SWPHES) and Underground PHES (UPHES);

- costs are reported and used for assessing economic feasibility of each EES technology in a realistic environment represented by an Italian case study. Based on this estimation, a recommendation on which technologies are the most the suited ones from the economic point of view is provided.

\section{EES technologies overview}

- Pumped hydro energy storage (PHES) plants are made up of an upper and a lower reservoir, which are connected by one or more penstocks. The storage is charged by pumping water and it is discharged by turbining water. It could seem that the recent interest for storage stems from the fact that easily exploitable sites suited for PHES are already depleted [2]. Actually, PHES has some major drawbacks like the large use of land area and the large impact on river course and flow rates. The use of sea water reservoirs and cliffs (SWPHES), has been proposed to overcome the lack of easily exploitable sites [5], whereas by using abandoned caves and mines as lower reservoirs [6] (UPHES), the environmental impact could be largely reduced. To date, 
only one SWPHES pilot plant has been built (30 MW facility in Okinawa, Japan [7]), but some other facilities are planned [5]. In UPHES, the higher reservoir may be built on top of the facility, thus using an already exploited terrains with no value. Furthermore, by using the water in closed loop, the environmental impact of the facility could be furtherly reduced.

- Compressed Air Energy Storage (CAES) stores electric energy by compressing environment air and by storing it under pressure. The air is then discharged and used to feed a gas turbine combustion chamber. Two CAES are currently in operation: Huntdorf (Germany), commissioned in 1969 and M clntosh in A labama (USA), commissioned in 1991 [8]. B oth facilities use natural gas to power up the turbine during the discharge phase. To cut the costs, salt domes, rocky caves and aquifers are proposed as pressurized air storages, but this makes CAES site dependent [8]. To solve this issue, Under Water CAES (UWCAES), which uses sea/lake water hydraulic head to pressurize the storage [9], has been proposed. To date, only one UWCAES pilot plant has been commissioned in Lake Ontario (Toronto, Canada) [8]. To avoid using fossil fuel, A diabatic CAES (ACAES) has been proposed. ACAES stores the excess thermal energy from the compression and use it to preheat air during discharge. The first pilot scale ACAES implementation $(500 \mathrm{~kW}, 4 \mathrm{~h})$ achieved a promising efficiency around 64\% [4], whereas the first grid scale facility $(90 \mathrm{MW}, 360 \mathrm{MWh}$ ) is planned for commissioning [4]. Heat recovery in ACAES can be also done by using water sprays and liquid piston compression/expansion to operate nearly isothermal transformations (Isothermal CAES: ICAES) [10].

- Liquified air energy storage (LAES) stores electric energy in form of cryogenic energy by liquefying air, which is stored and then pumped, heated and expanded in a turbine during discharge phase [7]. B asic LAES are based on Linde cycle, whereas most recent systems are based on Claude or Kapitza cycles. To date, only one LAES pilot plant (300 kW, $2.5 \mathrm{MWh}$ ) has been successfully commissioned [11]. Improvements to basic LAES comprise: internal regenerations and turbine outlet waste heat recovery with ORCS, Brayton cycles or Absorption cooling systems [12]. To cut energy consumption and costs, alternative fluids like $\mathrm{CO}_{2}$ have been proposed [13]. In fact, Liquified Carbon-dioxide Energy Storage (LCES) might be cheaper as it uses much more compact equipment. LAES has some advantages over CAES, like higher energy density and site independency. However, LAES generally achieves lower efficiency than CAES and PHES. This problem may be overcome by using non-standard configurations, which, however, might increase costs and start-up times, due to added thermal inertia.

- Sodium sulfur (NaS) battery energy storage use molten sodium $(\mathrm{Na}$ ) and molten sulfur $(\mathrm{S})$ as negative and positive electrodes. The two electrodes are divided by a solid electrolyte of $\beta$-alumina which allows for the ionic conduction. $\mathrm{NaS}$ batteries are commercially available and they count several $M W$ scale applications [14]. $\mathrm{N}$ aS batteries are cheap and their building materials are largely recyclable [4]. NaS batteries feature high energy density, fast response, long cycle life ( $>2500$ cycles at $80-100 \%$ DOD) and good efficiency [4]. NaS batteries operate between $290-350{ }^{\circ} \mathrm{C}$, to maintain electrodes liquid. During discharge phase, losses induced by internal resistance provide the necessary heat, whereas during idle or charge phase, the heat must be provided from the external and this may lead to parasitic losses up to $20 \%$ per day [15]. Cold start-up may take up to $15 h$ to allow electrodes to molten [15]. $\mathrm{N}$ aS batteries are very promising even though their use may be discouraged by electrodes corrosive behavior, by costs, which are higher than PHES and CAES ones, and by operating temperatures, which should be lower to operate more safely and with lower parasitic losses.

- Flow batteries use liquid electrolytes with dissolved active species, which are pumped through an electrochemical cell that converts chemical energy into electric energy. Electrolytes are stored in tanks, whose size determines battery capacity rating. Similarly, battery power rating is determined by the pumping capacity of the system. Therefore, capacity and power are decoupled, and this is a major advantages over traditional batteries $[4,14]$. Flow batteries have high efficiency, very quick response, very long cycle life and maximum discharge depth of $100 \%[4,7]$. On the other hand, flow batteries have high cost, low density and greater complexity if compared to other batteries. Flow batteries are commercially available (V anadium batteries) and they count several $M W$ scale applications [7]. One of the largest ( $25 \mathrm{MW}$ and $100 \mathrm{MWh}$ ) is in A stana (K azakhstan) [4].

- $\quad$ Pumped Thermal Electricity Storage (PTES) stores electric energy as thermal energy, which is later converted back into electricity. The charge is performed with heat pumps, whereas discharge phase is performed with heat engines. Thermal energy is stored as sensible or latent heat. B oth hot and cold reservoirs are used, but sometimes one is replaced by the environment, or by alternative heat sources/sinks. Compared to PHES and CAES, PTES usually has lower efficiency, but it is site independent. PTES technology may use both Brayton cycles (direct and inverse) and Rankine cycles (direct and inverse) [16]. Br-PTES most often uses packed bed sensible heat storage, usually made up of $\mathrm{Al}_{2} \mathrm{O}_{3}$, which has good conductivity/heat capacity ratio. Br-PTES is characterized by high energy density and very low estimated prices. Ra-PTES could be a valid alternative as it generally achieves higher densities and it stores energy at much lower temperature $\left(\sim 200{ }^{\circ} \mathrm{C}\right.$ vs. $\left.\sim 1000{ }^{\circ} \mathrm{C}\right)$, which may allow for the use of phase change materials and the integration of additional low-grade/waste thermal sources to improve efficiency [16].

A summary of the technical and economical features of the reviewed EES technology is reported in Table 1.

\section{EES economic feasibility analysis}

Based on Table 1 data, the economic feasibility of EES technology can be assessed. Calculations use the 2018 Italian hourly energy prices, taken from [17]. Storage optimal operation is calculated by means of a M ixed 
Table 1. EES technical and economic features. $\eta_{r t}$ : roundtrip efficiency; $\tau_{r}$ : response time; $\rho_{e n}$ : energy density; $L$ : operating life; $\Gamma_{p}$ : power cost; $\Gamma_{c}$ : capacity cost.

\begin{tabular}{|c|c|c|c|c|c|c|c|}
\hline Technology & $\Gamma_{p}[€ / \mathrm{kW}]$ & $\Gamma_{c}[€ / \mathrm{kWh}]$ & $\rho_{e n}\left[\mathrm{kWh} / \mathrm{m}^{3}\right]$ & $\tau_{r}[\mathrm{~min}]$ & $\eta_{r t}[\%]$ & $L[y]$ & References \\
\hline PHES & $400 / 600-1000 / 2000$ & $1 / 5-100$ & $0.5-1.5$ & $1-3$ & $65-85$ & $30-40+$ & {$[4,7,15,16,18]$} \\
\hline UPHES & $400 / 600-1000 / 2000^{a}$ & 85 & $0.5-1.5$ & $1-3$ & $70-85$ & $30-40$ & {$[6,15,16,18]$} \\
\hline SWPHES & $720-2200$ & $25-30$ & $0.3-0.4$ & $1-3$ & $70-85$ & $15+$ & {$[5,7]$} \\
\hline CAES & $400 / 500-800 / 1000$ & $1 / 2-50 / 100 / 200$ & $3-12$ & $10-15$ & $40-60$ & $20-40$ & {$[8,15,16,18]$} \\
\hline ACAES & $700-1000$ & $40-80$ & $0.5-20$ & $10-15$ & $60-70$ & $20-40$ & {$[4,15]$} \\
\hline UWCAES & $750-2000$ & $40-200$ & $0.3-5$ & $10-15$ & $60-70$ & 20 & {$[15,19]$} \\
\hline ICAES & $500-1000$ & $10-100$ & $1-25$ & $1-5$ & $60-80$ & $20-40$ & {$[8,15]$} \\
\hline LAES & $900 / 1000-2000$ & $260-530$ & 50 & $5-10$ & $50-60$ & $20-40$ & {$[4,15,16]$} \\
\hline Br-PTES & $600-800$ & $20 / 90-60 / 180$ & $110-200$ & $1-3$ & $50-70$ & $20-40$ & {$[15,16,20]$} \\
\hline Ra-PTES & $225^{b} / 390-450$ & $45-95 / 120$ & $140-170$ & $1-3$ & $40-60$ & $20-40$ & {$[15,21-23]$} \\
\hline N aS B att. & $150 / 200-300 / 900$ & $100 / 200 / 300-500 / 600$ & $150-250$ & $<0.005$ & $70-90$ & $10-15$ & {$[4,15,18]$} \\
\hline Flow B att. & $300 / 600-500 / 1500$ & $150 / 400-750 / 1000$ & $16-60$ & $<0.001$ & $60-85$ & $5-15$ & {$[4,15,18,24]$} \\
\hline
\end{tabular}

Integer Linear Programming (MILP) optimization problem. This allows to find the optimal sequence of storage charges and discharges to be performed each day in order to maximize the economic profit. Problem variables are discharge and charge power $P_{d / c}$ in each $i$-th hour of the day. Problem objective function is the net daily revenue $R_{\text {day }}$ (Eq.1):

$$
R_{\text {day }}=\sum_{i=1}^{24} P_{d, i} \cdot \pi_{i} \cdot \tau-\sum_{i=1}^{24} P_{c, i} \cdot \pi_{i} \cdot \tau
$$

where $\tau$ is equal to $1 h$ and $\pi$ is the hourly energy price in $€ / M W h$. Problem constraints are (Eq. 2):

$$
\begin{gathered}
0 \leq P_{d, i} \leq P_{n o m} \\
0 \leq P_{c, i} \leq P_{n o m} \\
0 \leq \tau \cdot \eta_{c} \sum_{n=1}^{i} P_{c, i, n}-\frac{\tau}{\eta_{d}} \sum_{n=1}^{i} P_{c, i, n}+C_{0} \leq C_{n o m} \\
\tau \cdot \eta_{c} \sum_{n=1}^{24} P_{c, i, n}-\frac{\tau}{\eta_{d}} \sum_{n=1}^{24} P_{c, i, n}=0
\end{gathered}
$$

where charge and discharge efficiency $\eta_{c}=\eta_{d}=\sqrt{\eta_{r t}}$ and $C_{0}$ is equal to 0 and $P_{\text {nom }}=10 \mathrm{MW}$ and $C_{\text {nom }}=40$ $M W h$ are the nominal dimensions of the analysed EES. The listed constraints ensure that the storage cannot charge/discharge more than what is allowed by its nominal power and capacity ratings and that the storage is emptied at the end of each day. Not all the storages have $\eta_{c}=\eta_{d}$ or minimum charge level equal to 0 . For the sake of simplicity, all the EES technologies are treated equally and these hypotheses are enough for first order estimation purposes. By summing the daily revenue over the year, the annual revenue $R$ is found. $R$ may be compared with annualized cost of storage $A$, which is calculated as in Eq. 3:

$$
A=\frac{r(1+r)^{L}}{(1+r)^{L}-1}\left(P_{n o m} \cdot \Gamma_{p}+C_{n o m} \cdot \Gamma_{c}\right) \cdot 1000
$$

where $r=0.07$ is the interest rate and $L$ is the storage operating life. Therefore, different EESs have different revenue, as they have different efficiencies, and different annualized costs, as they have different specific costs and operating lives. In Table 1 a range is reported for $\eta_{r}$, $L, \Gamma_{p}$ and $\Gamma_{c}$, then a range for $A$ and $R$ may be defined as well. The Extrema of these ranges represent the best and the worst cases for these quantities. In addition to these, an average value, calculated with the average of each range is investigated. The results regarding the comparison between $A$ and $R$ are reported in Figure 1, where three cost scenarios are reported: base case (Eq 3 ), $25 \%$ discount and $50 \%$ discount. This allows to consider the impact of price reductions or the effect of subsidies. Several conclusions may be drawn from Figure 1:

- None of the listed technologies achieved parity between $A$ and $R$, not even in the case of $50 \%$ cost reduction. Therefore, none of the listed technologies is currently economically viable, in the selected case study;

- All the technologies have $A$ that is around one order of magnitude higher than $R$. Therefore, the listed technologies are far from economic feasibility;

- The higher the efficiency, the higher the revenue, therefore batteries should be advantaged. However, since the incomes are well under the required ones, Flow and $\mathrm{N}$ aS batteries are actually among the worst performing due to their high costs. This demonstrates that cost and operating life may be more impactful than efficiency, in the context of grid scale load shifting. In other words, the $\mathrm{NaS}$ and Flow batteries cost is disproportioned, if compared to benefits brought by their efficiency;

- $\quad$ by calculating to $A_{\text {ave }}$ and $R_{\text {ave }}$ difference, the most promising technologies can be determined; they are RaPTES, ICAES, ACAES and Br-PTES and this justifies the research interest towards these alternative EES. On the other hand, the least promising technologies, i.e. those that have the larger difference between $A_{\text {ave }}$ and $R_{\text {ave, }}$ are Flow batteries, NaS batteries and LAES.

Based on Figure 1 results, it may be interesting to calculate how much the energy prices should change to make the EES technologies able to repay themselves. To do this, it can be observed that daily revenue $R_{\text {day }}$ may be modelled as in Eq. 4:

$R_{\text {day }} \approx R_{\text {model }}=\max \left[0 ; \beta \cdot\left(\eta_{r t} \cdot \pi_{d}-\pi_{c}\right)\right]$

where $\beta$ is an empirical constant to be fitted from data, and $\pi_{c, d}$ are the average energy prices at which the energy is charged and discharged. The data for $\beta$ estimation come from the solution of $\mathrm{Eq} .1$ and 2optimization problem, whereas $\pi_{c}$ and $\pi_{d}$ are calculated by averaging the lowest and highest four $\pi_{i}$ values for each day. This may be justified by considering that the storage has a power/capacity ratio equal to $1 / 4 h^{-1}$, therefore it charges and discharges for approximately four hours per day. 


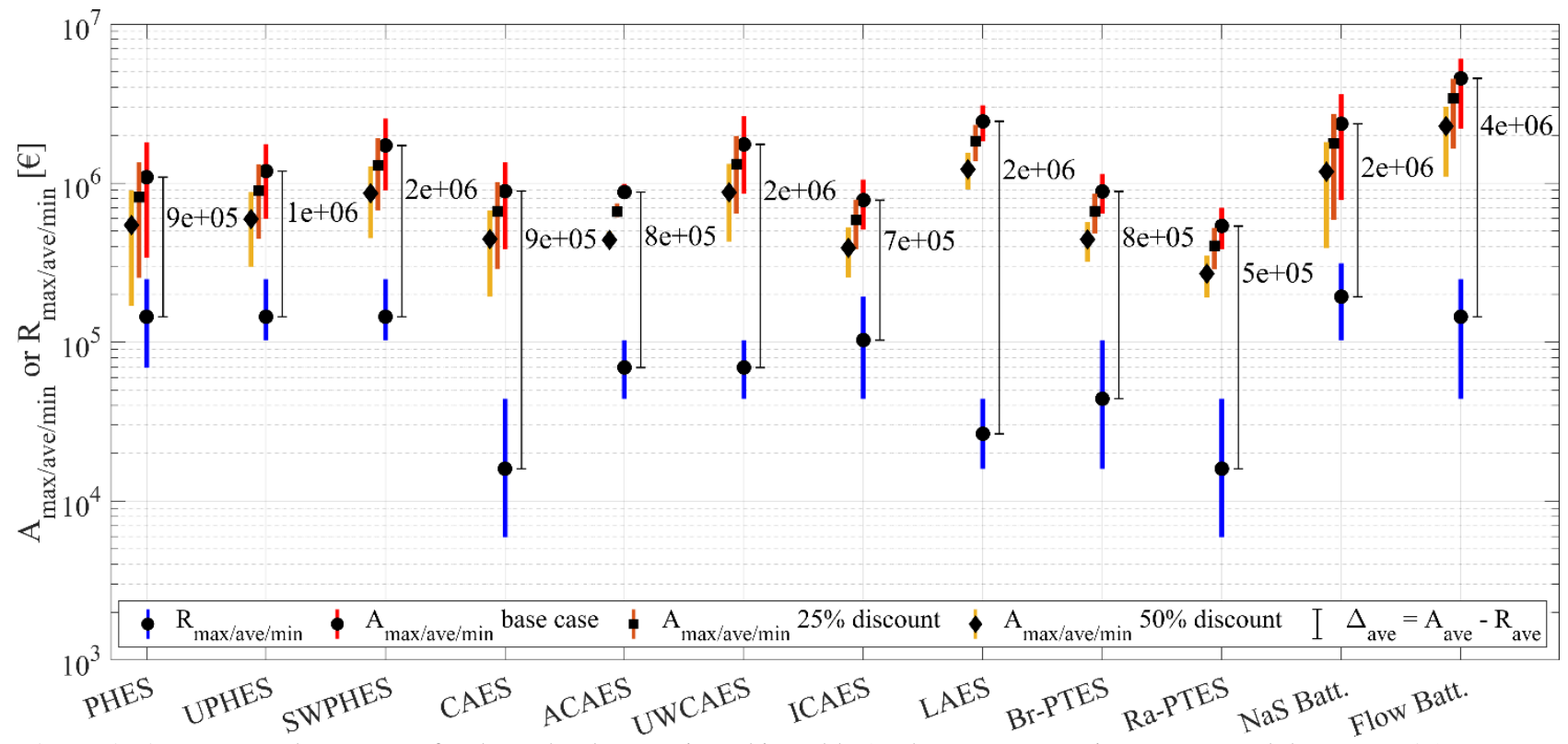

Figure 1. $A_{\max / a v e / m i n}$ and $R_{\max / a v e / m i n}$ for the technology reviewed in Table 1. Three cost scenarios are reported: base case, $25 \%$ discount and $50 \%$ discount.

The results related to the fit of the Eq. 4 model are reported in Figure 2, where it is shown how the model can explain reasonably well the daily revenue values.

By using $R_{\text {model }}$, the $\pi_{d}$ and $\pi_{c}$ values that yield the current average daily revenue may be calculated from Eq. 5:

$$
R_{\text {mean }}=\frac{\sum_{j=1}^{365} R_{\text {day,j }}}{365}=\beta \cdot\left(\eta_{r t} \cdot \pi_{d}-\pi_{c}\right)
$$

Similarly, the $\pi_{d}$ and $\pi_{c}$ values that yield a daily average revenue, such that the annual revenue is equal to the annuity $A_{\text {ave }}$, may be calculated from Eq. 6:

$$
A_{\text {ave }}=\frac{\beta \cdot\left(\eta_{r t} \cdot \pi_{d}-\pi_{c}\right)}{365}
$$

If $\pi_{c}$ is set, two val ues of $\pi_{d}$ that satisfies respectively Eq. 5 and 6 may be found. These values are function of $\eta_{r t}$, then three cases for each may be defined according to maximum, average and minimum $\eta_{r t}$ values from Table 1. By comparing the two resulting $\pi_{d}$ values from Eq. 5 and 6 , the price changes required to make the reviewed EES technology viable may be estimated. From Figure 1, it is already known which technology are the most promising ones from the economic point of view. In this regard, by calculating $\pi_{d}$ a different estimation may be done. In fact, $\pi_{d}$ values from Eq. 5 give an idea of what are the current price situation on the Italian market. On the other hand, $\pi_{d}$ values from Eq. 6 provide an estimation of what should be the peak prices on the average for the market to being able to sustain the EES. Of course, in market situations with a strong shortage of dispatchable production, very high prices may arise. N onetheless, these extreme scenarios may be avoided, or discouraged, by system operators by means of economic incentives, like Capacity $M$ arkets, or others. Therefore, it is unclear what $\pi_{d}$ values may be realistically achieved. Furthermore, very high $\pi_{d}$ values will not suddenly appear on the markets, as the RES development is a slow and steady process. This might be a problem for storage development, as the required economic driver might be absent in all the intermediated steps that divide the current situation from a hypothetical high-RES penetration scenario. A vicious circle might arise, in which storage might be feasible only if a large share of productive capacity is represented by non-dispatchable RESs, but RESs cannot develop up to that point because they would need storage to foster their integration. From this point of view, the fact that none of the current technologies might be economically feasible is very concerning, since it may hinder the RES development and, with this, the power production sector decarbonization.

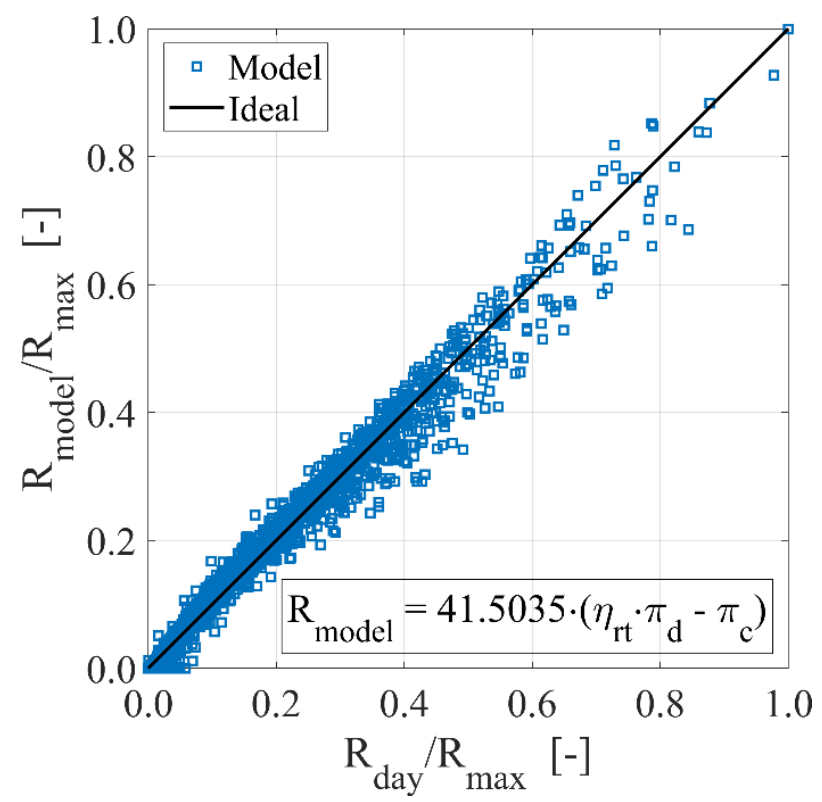

Figure 2. Parity plot between Eq. 4 model $R_{\text {model }}$ and daily revenue $R_{\text {day }}$ calculated by solving the optimization problem stated in Eq. 1 and 2. 


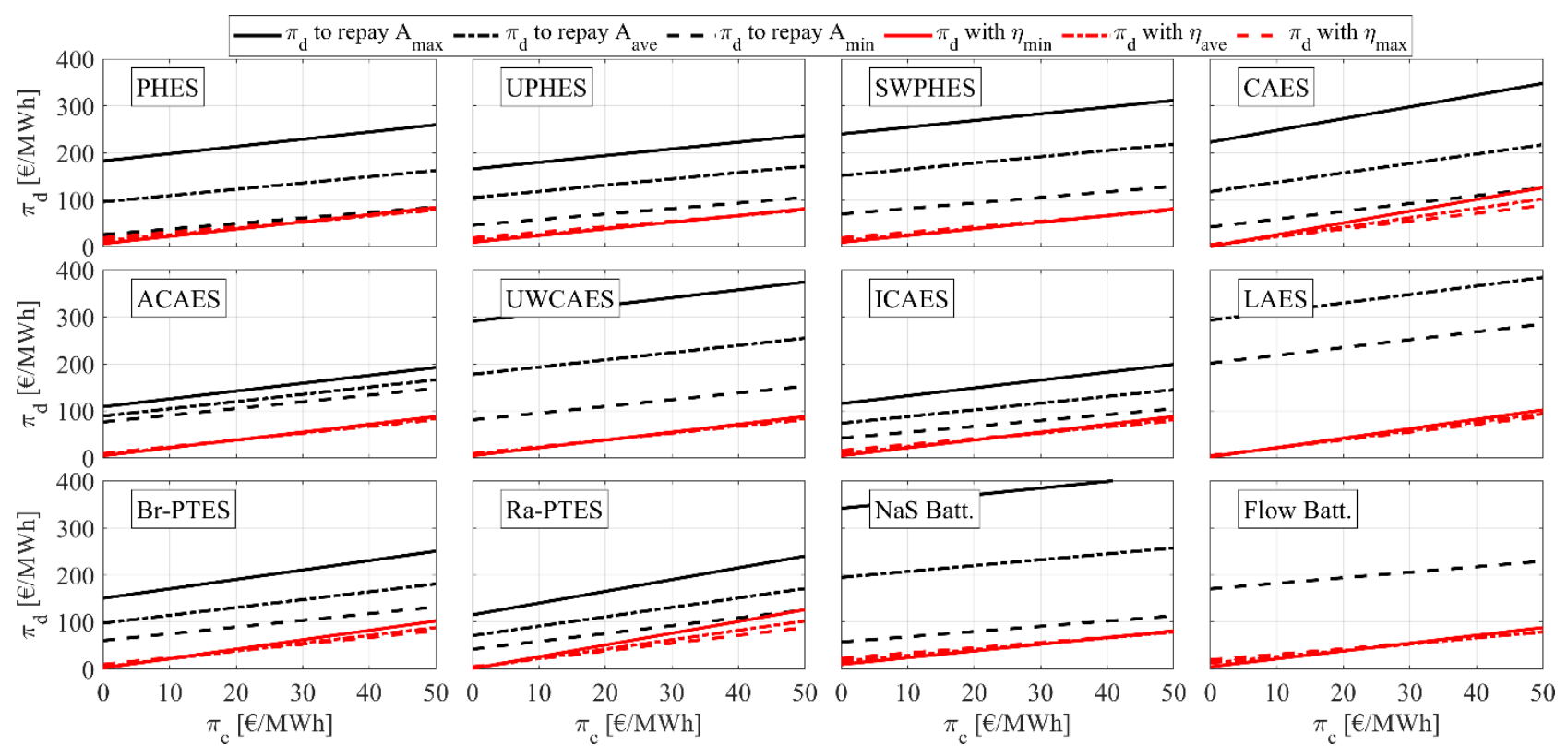

Figure 3. $\pi_{d}$ as a function of $\pi_{c}$ for three values of $\eta_{r t}$ ( $\left.\mathrm{min} / \mathrm{max} / \mathrm{ave}\right)$ and three values of $A$ ( $\left.\mathrm{min} / \mathrm{max} / \mathrm{ave}\right)$ for each technology of Table 1. $\pi_{d}$ values that satisfy Eq. 5 are in red, whereas $\pi_{d}$ values that satisfy Eq. 6 are in black.

The results related to the calculation of $\pi_{d}$ through Eq. 5 and 6 are reported in Figure 3 . A very interesting case is that with $\pi_{c}=0$. As a matter of fact, in a high RESpenetration scenario, charge would occur in the moment of maximum RES production. Due to the large overcapacity required to power up a nation-wide electric system al most with RESs, the demand should not be able to accommodate all the produced energy during production peaks. Therefore, in these moments, energy prices will be very low and even negative, if allowed to be so. Since negative prices are common only in few energy markets, this possibility is disregarded here, and the minimum considered charging price is equal to 0 .

With $\pi_{c}=0$ and $A_{\min }$, all the reviewed technology, except Flow batteries and LAES, can repay their own costs with an average $\pi_{d}<100 € / M W h$. Even in the case of $\pi_{c}=0$ and $A_{\text {ave }}$, some technologies are still able to repay themselves with an average $\pi_{d}$ slightly lower than 100 $€ / M W h$. These technologies are: PHES, UPHES, ACAES, ICAES, Br-PTES and Ra-PTES. In this case, $\mathrm{NaS}$ battery would require an average $\pi_{d}$ equal to 200 $\epsilon / M W h$. Finally, for the third case, i.e. $\pi_{c}=0$ and $A_{\max }$, few technologies should be able to sustain they self with a minimum $\pi_{d}$ lower than $150 € / M W h$. These technologies are: ACAES, ICAES, Br-PTES and RaPTES, thus confirming the strong potentiality of these technologies, based on different operating principles than batteries.

It is worth noting that energy prices around $100-150$ $\epsilon / M W h$ should not be considered unrealistically high, as they are currently occurring in several energy market, the Italian one included. However, such prices are not common nowadays, therefore storages cannot gain enough to self-sustain in current market scenarios.

The resulting $\pi_{d}$ values may be considered as compatible with realistic future market and energy mix scenarios. Therefore, some EES technologies like PTES, some forms of CAES and some of PHES may realistically become viable in the future. Other technologies like $\mathrm{NaS}$ and Flow batteries might encounter much more difficulties in achieving the economic feasibility, even in very positive scenarios.

Finally, as Figure 3 reports, $\pi_{d}$ is linear with $\pi_{c}$, therefore all the discussed price threshold should be adjusted, if other values of $\pi_{c}$ are considered. For example, if $\pi_{c}=10$ $\epsilon / M W h$ the only technologies that could achieve the economic parity in the worst-case scenario $\left(A_{\max }\right)$, without surpassing the threshold of $\pi_{d}=150 € / M W h$ are: ACAES, ICAES and Ra-PTES.

\section{Conclusive remarks}

In this paper a review of EES technologies suited for load shifting was performed. As a result of the review, a range of values for power and capacity costs, roundtrip efficiencies and operating lifetimes were defined for each technology. These data were used to assess the economic feasibility of the reviewed technologies in a realistic European case study (I talian 2018 hourly energy prices). As it resulted, none of the reviewed technologies is currently feasible from an economic point of view and often the annualized costs are of one order of magnitude higher than the revenues.

Based on these results, a simplified model that links daily revenues with the roundtrip efficiency and the charging and discharging energy prices is defined and tuned. By means of this model, the average energy prices required for covering the annualized costs were calculated in function of the charging energy prices.

For low and very low charging energy prices, the most promising technologies resulted to be ACAES, ICAES and Ra-PTES, followed by Br-PTES, PHES and UPHES. All these technologies might become viable with average maximum daily energy prices lower than $150 € / M W h$. Such values are higher than the current energy prices, but they might be realistically achieved for future energy mixes, as similar prices already occur in few days per year even today. 


\section{References}

[1] J. Devlin, K. Li, P. Higgins, A. Foley, Gas generation and wind power: $\mathrm{A}$ review of unlikely allies in the United Kingdom and Ireland, Renew. Sustain. Energy Rev. 70 (2017) 757768. doi:10.1016/j.rser.2016.11.256.

[2] M. Gimeno-Gutiérrez, R. Lacal-Arántegui, Assessment of the European potential for pumped hydropower energy storage based on two existing reservoirs, Renew. Energy. 75 (2015) 856-868. doi:10.1016/j.renene.2014.10.068.

[3] IEA, W orld Energy Outlook 2018, Paris, 2018.

[4] M.C. Argyrou, P. Christodoulides, S.A. Kalogirou, Energy storage for electricity generation and related processes: Technologies appraisal and grid scale applications, Renew. Sustain. Energy Rev. 94 (2018) 804-821. doi:10.1016/j.rser.2018.06.044.

[5] D.A. Katsaprakakis, D.G. Christakis, I. Stefanakis, P. Spanos, N. Stefanakis, Technical details regarding the design, the construction and the operation of seawater pumped storage systems, Energy. 55 (2013) 619-630. doi:10.1016/j.energy.2013.01.031.

[6] W.F. Pickard, The history, present state, and future prospects of underground pumped hydro for massive energy storage, Proc. IEEE. 100 (2012)

$473-483$. doi:10.1109/JPROC.2011.2126030.

[7] X. Luo, J. Wang, M. Dooner, J. Clarke, Overview of current development in electrical energy storage technologies and the application potential in power system operation, Appl. Energy. $\quad 137 \quad$ (2015) 511-536. doi:10.1016/j. apenergy.2014.09.081.

[8] M. Budt, D. Wolf, R. Span, J. Y an, A review on compressed air energy storage: Basic principles, past milestones and recent developments, Appl. Energy. $\quad 170 \quad$ (2016) 250-268. doi:10.1016/j.apenergy.2016.02.108.

[9] A.J. Pimm, S.D. Garvey, M. de Jong, Design and testing of Energy Bags for underwater compressed air energy storage, Energy. 66 (2014)

496-508. doi:10.1016/j.energy.2013.12.010.

[10] W. He, J. Wang, Optimal selection of air expansion machine in Compressed Air Energy Storage: A review, Renew. Sustain. Energy Rev. 87 (2018) 77-95. doi:10.1016/j.rser.2018.01.013.

[11] R. Morgan, S. Nelmes, E. Gibson, G. Brett, Liquid air energy storage - A nalysis and first results from a pilot scale demonstration plant, A ppl. Energy. 137 (2015) 845-853. doi:10.1016/j.apenergy.2014.07.109.

[12] A. Tafone, E. Borri, G. Comodi, M. van den Broek, A. Romagnoli, Liquid Air Energy Storage performance enhancement by means of Organic Rankine Cycle and Absorption Chiller, A ppl. Energy. 228 (2018) 1810-1821. doi:10.1016/j.apenergy.2018.06.133.

[13] M. Wang, P. Zhao, Y. Yang, Y. Dai, Performance analysis of energy storage system based on liquid carbon dioxide with different configurations, Energy. 93 (2015) 1931-1942. doi:10.1016/j.energy.2015.10.075.

[14] B. Dunn, H. Kamath, J.-M. Tarascon, Electrical Energy Storage for the Grid: A Battery of Choices, Science (80-. ). 334 (2011) 928-935. doi:10.1126/science.1212741.

[15] A.B. Gallo, J.R. Simões-M oreira, H.K.M. Costa, M.M. Santos, E. Moutinho dos Santos, Energy storage in the energy transition context: A technology review, Renew. Sustain. Energy Rev. 65 (2016) 800-822. doi:10.1016/j.rser.2016.07.028.

[16] A. Benato, A. Stoppato, Pumped Thermal Electricity Storage: A technology overview, Therm. Sci. Eng. Prog. 6 (2018) 301-315. doi:10.1016/j.tsep.2018.01.017.

[17] GME (Italian energy market operator), Italian Day-ahead energy market prices, (2019). https://www.mercatoel ettrico.org/it/download/D atiStorici.aspx (accessed September 1, 2019).

[18] B. Zakeri, S. Syri, Electrical energy storage systems: A comparative life cycle cost analysis, Renew. Sustain. Energy Rev. 42 (2015) 569 596. doi:10.1016/j.rser.2014.10.011.

[19] A.J. Pimm, S.D. Garvey, M. de Jong, Design and testing of Energy Bags for underwater compressed air energy storage, Energy. 66 (2014) 496-508. doi:10.1016/j.energy.2013.12.010.

[20] A. Smallbone, V. Jülch, R. Wardle, A.P. Roskilly, Levelised Cost of Storage for Pumped Heat Energy Storage in comparison with other energy storage technologies, Energy Convers. $\begin{array}{llll}M & 152 & \text { (2017) 221-228. }\end{array}$ doi:https://doi.org/10.1016/j.enconman.2017.09. 047.

[21] S. Staub, P. Bazan, K. Braimakis, D. Müller, C. Regensburger, D. Scharrer, B. Schmitt, D. Steger, R. German, S. K arellas, M. Pruckner, E. Schlücker, S. Will, J. Karl, Reversible heat pump-organic rankine cycle systems for the storage of renewable electricity, Energies. 11 (2018). doi:10.3390/en11061352.

[22] M. Morandin, M. Mercangöz, J. Hemrle, F. Maréchal, D. Favrat, Thermoeconomic design optimization of a thermo-electric energy storage system based on transcritical $\mathrm{CO} 2$ cycles, Energy. $58 \quad$ (2013) 571-587. doi:10.1016/j.energy.2013.05.038.

[23] S. Henchoz, F. B uchter, D. Favrat, M. M orandin, M. Mercangöz, Thermoeconomic analysis of a solar enhanced energy storage concept based on thermodynamic cycles, Energy. 45 (2012) 358365. doi:10.1016/j.energy.2012.02.010.

[24] D.H. Doughty, P.C. Butler, A.A. A khil, N.H. Clark, J.D. Boyes, Stationary Electrical Energy Storage, Electrochem. Soc. Interface. 19 (2010) 49-53. 